\title{
Whole breast proton irradiation for maximal reduction of heart dose in breast cancer patients
}

\author{
Mirjam E. Mast • Eline J. Vredeveld · Herman M. Credoe · Jaap van Egmond • \\ Mark W. Heijenbrok · Eugen B. Hug • Patrick Kalk • Loes M. L. van Kempen-Harteveld • \\ Erik W. Korevaar · Hans Paul van der Laan · Johannes A. Langendijk · \\ Hans J. E. Rozema · Anna L. Petoukhova · Jacobus M. Schippers • \\ Henk Struikmans $\cdot$ John H. Maduro
}

Received: 17 June 2014/ Accepted: 20 September 2014/Published online: 1 October 2014

(C) The Author(s) 2014. This article is published with open access at Springerlink.com

\begin{abstract}
Purpose In left-sided breast cancer radiotherapy, tangential intensity modulated radiotherapy combined with breath-hold enables a dose reduction to the heart and left anterior descending (LAD) coronary artery. Aim of this study was to investigate the added value of intensity modulated proton therapy (IMPT) with regard to decreasing the radiation dose to these structures. Methods In this comparative planning study, four treatment plans were generated in 20 patients: an IMPT plan and a tangential IMRT plan, both with breath-hold and free-breathing. At least $97 \%$ of the target volume had to be covered by at least $95 \%$ of the prescribed dose in all cases. Specifically with respect to the heart, the LAD, and the target volumes, we analyzed the maximum doses, the mean doses, and the volumes receiving
\end{abstract}

M. E. Mast $(\bowtie) \cdot$ J. van Egmond .

L. M. L. van Kempen-Harteveld · H. J. E. Rozema ·

A. L. Petoukhova $\cdot$ H. Struikmans

Radiotherapy Centre West, Lijnbaan 32, 2501 CK The Hague,

The Netherlands

e-mail: m.mast@mchaaglanden.nl

E. J. Vredeveld · H. M. Credoe · P. Kalk

E. W. Korevaar · H. P. van der Laan - J. A. Langendijk ·

J. H. Maduro

Department of Radiation Oncology, University Medical Center

Groningen, University of Groningen, Hanzeplein 1,

9713 GZ Groningen, The Netherlands

M. W. Heijenbrok

Department of Radiology, Medical Center Haaglanden, Lijnbaan

32, 2501 CK The Hague, Netherlands

E. B. Hug

ProCure Proton Therapy Centers, 103 Cedar Grove Lane,

Somerset, NJ 08873, USA

J. M. Schippers

Paul Scherrer Institut, 5232 Villigen PSI, Switzerland
5-30 Gy. Results As compared to IMRT, IMPT resulted in significant dose reductions to the heart and LAD-region even without breath-hold. In the majority of the IMPT cases, a reduction to almost zero to the heart and LAD-region was obtained. IMPT treatment plans yielded the lowest dose to the lungs. Conclusions With IMPT the dose to the heart and LAD-region could be significantly decreased compared to tangential IMRT with breath-hold. The clinical relevance should be assessed individually based on the baseline risk of cardiac complications in combination with the dose to organs at risk. However, as IMPT for breast cancer is currently not widely available, IMPT should be reserved for patients remaining at high risk for major coronary events.

Keywords Breath-hold - Breast cancer - IMPT - IMRT · Heart sparing

\section{Introduction}

Postoperative radiotherapy is considered standard of care after breast-conserving surgery for breast cancer [1]. After mastectomy, radiotherapy is required in case of intermediate or high risk of locoregional failure [2, 3]. Previous studies [4, 5] have shown that radiotherapy is associated with an increased rate of major coronary events, especially in patients treated for left-sided breast cancer. However, it should be noted that the follow-up period in these studies is relatively short $[4,5]$. With improved survival, more patients will be at risk for long-term radiation-induced toxicity, thus making it even more important to reduce the dose to all organs at risk (OARs).

Recently, Darby et al. found that the rate of major coronary events was proportional to the mean dose to the heart starting within a few years after exposure. Patients with pre-existing cardiac risk factors had higher absolute 
risks after radiotherapy than those without [6]. Given its anatomical location, the left anterior descending (LAD) coronary artery is most at risk for developing atherosclerosis after left-sided breast-conserving radiotherapy [7]. Taylor et al. showed that even with contemporarily delivered tangential fields, the mean dose to the LAD was considerable: $7.6 \mathrm{~Gy}$. Furthermore, half of the patients appeared to receive more than $20 \mathrm{~Gy}$ in the ventral part of the heart [8]. As the rate of ischemic heart disease is proportional to the mean heart dose, Darby et al. advised to reduce the dose to the heart as much as possible. In order to reduce the dose to the heart and the LAD using photons, intensity modulated radiotherapy (IMRT), either combined or not combined with breath-hold techniques, has been investigated [9-11] and compared to 3D-conformal radiotherapy (3D-CRT) with and without breath-hold [11].

A commonly used IMRT technique for breast cancer treatment is an IMRT technique based on the standard tangential fields with additional smaller subfields in order to improve dose homogeneity [12]. The advantage of this technique, compared to the full inversed planned multiple beam IMRT, is, that the dose redistribution is confined to the same area as the tangential fields, thus avoiding an excessive low dose to surrounding OARs. In addition, breath-hold techniques can be used to decrease the heart dose. With a breath-hold technique, a patient holds her breath during $25-30 \mathrm{~s}$ intervals in which radiation is administered. In doing so, the distance between the heart and the radiation fields increases and, consequently, the dose to the heart decreases [10].

However, due to anatomical variations in some patients, the radiation dose to the heart remains relatively high, even with the use of advanced photon-based techniques. Due to its physical characteristics, proton therapy may eventually enable a further decrease of dose to the heart. In contrast to a photon beam, a proton beam is characterized by a very narrow width of a relatively high peak of maximum dose administration: the Bragg peak. In other words, a proton beam is characterized by a dose distribution that is finite and adjustable in depth depending on the energy of the proton beam. Theoretically, these characteristics of protons enable a very precise irradiation of the target volume, while at the same time better sparing of the surrounding normal tissue can be obtained [13].

Therefore, we assumed that proton therapy may enable an improved sparing of the heart and LAD in left-sided breast cancer patients, especially in cases where the heart dose remains (relatively) high with advanced photon techniques [14-16]. In a previous paper, we found that tangential IMRT in combination with a breath-hold procedure resulted in a significant decrease of the dose to the heart and LAD-region compared to 3D-CRT in breathhold, while retaining optimal target volume coverage [11].
Furthermore, compared to standard photon 3D-CRT, tangential IMRT improves overall cosmesis and reduces the risk of skin telangiectasia [17]. However, to the best of our knowledge, planning comparative studies are lacking, which focus on the additional value of protons for whole breast irradiation compared to that of tangential IMRT (both with and without breath-hold).

Therefore, the aim of this planning comparative study was to determine whether a further dose reduction to the heart and LAD could be obtained with proton therapy (either with or without breath-hold).

\section{Methods}

We used the same methods as described in our previous planning comparative study comparing conformal photon radiotherapy (3D-CRT) and tangential IMRT (with and without breath-hold) [11]. The current study population consisted of 20 consecutive female breast cancer patients (pT1-2; pN0-1; M0). All patients underwent breast-conserving surgery and axillary staging with a sentinel node procedure.

To avoid interobserver-based delineation differences, the glandular breast tissue was contoured by one experienced radiation oncologist (LKH), according to RTOG delineation guidelines [18], and defined as the CTV. The PTV was created by expanding the CTV with $5 \mathrm{~mm}$ in transverse directions, $6 \mathrm{~mm}$ cranially, and $9 \mathrm{~mm}$ caudally according to the guidelines of our department for 3D-CRT and IMRT. The PTV was retracted $5 \mathrm{~mm}$ from the patient surface $\left(\mathrm{PTV}_{\text {trim }}\right)$ to minimize high-dose levels in the buildup regions for IMRT plans. No adaptations for PTV $_{\text {trim }}$ were performed in the direction of the lungs, in doing so the thoracic wall may be included in $\mathrm{PTV}_{\text {trim. }}$. In order to be able to compare the same volumes, we applied the same margins to the proton plans. Furthermore, the heart and the LAD-region were delineated by one experienced radiation oncologist (LKH) and were subsequently reviewed by an experienced cardiac radiologist $(\mathrm{MH})$. All volumes were delineated on each breath-hold scan and free-breathing scan. For the breath-hold scan, the active breathing control (ABC) method was used (ELEKTA Active Breathing Coordinator $^{\mathrm{TM}}$ device, Crawley, United Kingdom) [19]. A high feasibility rate was reported when using the $\mathrm{ABC}$ method $[10,20]$. Details concerning the ABC method were described by Mast et al. [11].

Treatment planning techniques

\section{Tangential IMRT-planning}

All IMRT plans were produced by one experienced dosimetrist (HR), who was blinded for the IMPT plans. The 
applied IMRT technique was a tangential IMRT technique. According to this technique, approximately $60 \%$ of the dose was given with two tangential open fields, and $40 \%$ with four inversely planned tangential IMRT fields using the same gantry angles, with a 'step-and-shoot' technique $[11,12]$. The nominal energy used was $6 \mathrm{MV}$ in most of the cases, and occasionally $10 \mathrm{MV}$.

\section{Proton planning}

Spot scanning intensity modulated proton therapy (IMPT) plans were planned by two experienced IMPT dosimetrists (HC, PK) using a research version of the Pinnacle3 planning system (version 9.1, Philips Medical Systems, United States). Both were blinded for the IMRT plans. With spot scanning, a pencil beam of protons is regulated in a highdose spot. This spot can be positioned for a specified period of time; by superimposing several spots, the desired radiation dose can be composed. Generally, for protons a RBE of 1.1 is used over the full depth of the proton beam, and the dose is represented as CGE (cobalt gray equivalent, which is RBE $\times$ physical dose in Gy) [15]. In the doses we report here, this $\mathrm{RBE}$ has been taken into account.

IMPT dose calculations and field configurations were planned according to Ares et al. [21]. In all plans, the gantry angles were $345^{\circ}\left(-15^{\circ}\right), 27^{\circ}$, and $75^{\circ}$. The different beams were set to distribute the spots in such a way that no spot was more than $0.2 \mathrm{~cm}$ outside the $\mathrm{PTV}_{\text {trim. }}$. Spots were placed over the PTV $\mathrm{PTim}_{\text {trim }}$ with $\mathrm{mm}$ separation in the plane perpendicular to the beam direction; while in depth, spot layers were positioned and interspaced with $5 \mathrm{~mm}$ between each spot.

Energy layers ranged from 7.7 to $23.0 \mathrm{~g} / \mathrm{cm}^{2}$ (representing the depth of the Bragg peak location) or 100-185 MeV. Corresponding lateral spot sizes ranged approximately from 15 to $8 \mathrm{~mm}$ full-width-at-half-maximum at the isocenter in air and without range shifter. A range shifter of $75 \mathrm{~mm}$ water equivalent thickness was used so that the spot positions ranged from 2 to $155 \mathrm{~mm}$ water equivalent depth. Note that the range shifter and air gap between range shifter and patient skin increase the spot size.

All plans were adapted to the individual target volumes and critical organs, using the "trial-and-error" method.

\section{IMRT and IMPT treatment plan optimization}

The prescribed dose was $42.56 \mathrm{~Gy}$ in 16 fractions in all cases. For all IMRT and IMPT plans, $97 \%$ of the PTV trim $_{\text {IM }}$ had to be covered by at least $95 \%$ of the prescribed dose with a maximum of $2 \%$ receiving more than $107 \%$ of the prescribed dose [22]. No compromises on the PTV coverage with either of the techniques were made to ensure a fair comparison. For the $\mathrm{PTV}_{\text {trim, }}$ the following constraints were used: uniform dose $(42.56 \mathrm{~Gy})$, maximum dose (45.5 Gy, point dose), and minimum dose (40.6 Gy). The maximum dose $\left(D_{\max }\right)$ was defined as the maximal dose to a volume of at least $2 \%$ of that specific volume; according to the ICRU 83. All further planning objectives used were similar again to obtain fair dosimetric comparisons between the two techniques. For the purposes of our study, IMRT and IMPT treatment plans based on the breath-hold and free-breathing scans were compared in all patients. Furthermore, various dose volume parameters of PTV $\mathrm{Prim}_{\text {, }}$, heart, LAD-region, and lung (both lungs as well as the left lung separately) were generated and evaluated. The choice of these dose volume parameters ( $D_{\max }$; mean; V5V30 Gy) was based on those published in the literature [10, 22, 23]. Finally, all plans were evaluated and approved by two experienced breast cancer radiation oncologists (HS and JHM).

\section{Statistics}

A Wilcoxon signed-rank test was performed to compare dose and volume differences since the number of eligible cases was less than 30. For this analysis, we used SPSS Statistics version 20.0. The level of statistical significance was defined by a $p$ value of $\leq 0.05$ (two-sided) for all tests.

\section{Results}

\section{Heart and LAD-region}

The mean doses for the heart and LAD-region, for IMRT and IMPT, in breath-hold and free-breathing, in all cases are presented in Fig. 1.

Despite the use of tangential IMRT with breath-hold in some patients, the dose to the LAD-region remained relatively high (Table 1; Fig. 1). With breath-hold IMRT, still 9 out of 20 patients received a mean dose to the LADregion exceeding $5 \mathrm{~Gy}$, while in 4 out of 20 patients the dose remained beyond 10 Gy. In 3 patients, the mean heart dose was more than 2 Gy (Fig. 1).

An additional reduction of the various dose parameters could be obtained with IMPT as well as with breath-hold IMPT. The volume of the heart and LAD-region receiving 20 Gy (V20 Gy) could be reduced to almost zero in all patients (Figs. 1, 2; Table 1).

Lung

As compared to IMRT, the mean lung dose, the V5 Gy, and the V20 Gy in both lungs and in the left lung could be reduced significantly. In particular, the mean V20 Gy value 


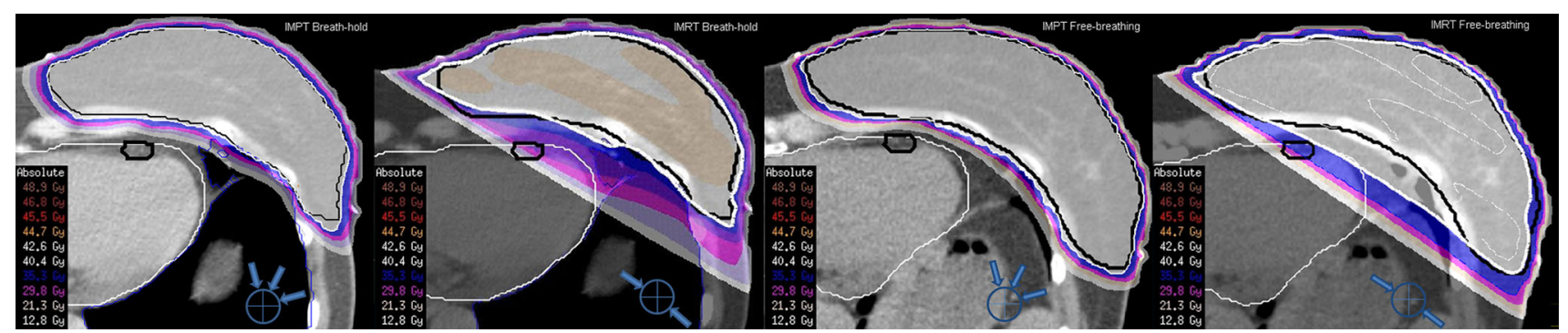

Fig. 1 Isodose lines in the caudal part of the patient on the breathhold scan. Delineated organs at risk: white line heart; black line region of the left anterior descending coronary artery. Planning target

for both lungs could be reduced from $5.1 \%$ (SD 2.2) with breath-hold IMRT to $1.3 \%$ (SD 0.8) with breath-hold IMPT (Table 1).

\section{Discussion}

The main objective of this study was to investigate if the dose to the heart and LAD-region could be reduced using spot scanning IMPT. The results showed that, with both IMPT techniques (with and without breath-hold), the doses to the heart as well as to the LAD-region could be reduced significantly compared to IMRT with breath-hold. This could be achieved without compromising the doses to the target volumes. It should be stressed that, with IMPT, a further reduction to almost zero to the heart and LADregion could be obtained in the majority of cases. The results show that a breath-hold technique had no added value when using IMPT. However, using breath-hold may improve the robustness of the IMPT technique, since the tissue shift will be less in breath-hold. Protons are more sensitive than photons to the effects of motion due to the range of the Bragg Peak. When using a proton field from a perpendicular direction, a tissue shift could cause thickness changes and thus range changes.

Recently Darby et al. reported a dose-effect relationship between the dose to the heart and the rate of major coronary events [6]. The authors could not identify any threshold dose for the development of coronary events, emphasizing the need to reduce the dose to as low as possible. The average mean heart dose of the left-sided breast cancer patients in their cohort was 6.6 Gy [6]. However, we noted lower mean heart doses with our tangential IMRT (2.7 Gy with free-breathing and 1.5 Gy with breath-hold). With IMPT further reductions could be obtained $(0.2 \mathrm{~Gy}$ with free-breathing and $0.1 \mathrm{~Gy}$ with breath-hold).

Our study compares two techniques using the same fractionation scheme, with a fraction dose of $2.66 \mathrm{~Gy}$ and a total dose of $42.65 \mathrm{~Gy}$. However, if the effects on reduction volume: black line $\mathrm{PTV}_{\text {trim }}$; thick white line $95 \%$ isodose line. At the bottom right, the used gantry angles were pointed out, represented by the small arrows

in cardiac dose of this study are being compared to the results of other planning studies, this needs to be taken into account.

It has been shown that decreasing of the mean heart dose is relevant [6]. The lifetime risk of radiation-induced ischemic heart disease for breast cancer patients increases linearly with an increase of the mean dose to the heart of $7.4 \%$ per Gy (95\% confidence interval, 2.9-14.5) [6]. Consequently, the baseline risk should be taken into account. Recently, Duma et al. [24] approximated the increased rate of absolute radiation-induced ischemic heart disease by using the tables of the Darby publication [6]. They reported that, irradiating a 50-year-old breast cancer patient without cardiac risk factors with a mean heart dose of $3 \mathrm{~Gy}$, the risk of having at least one acute coronary event by the age of 80 years rises from 4.5 to $5.4 \%$. They subsequently noted that in the presence of pre-existent cardiac risk factors, the risk of having at least one acute coronary event by the age of 80 years would rise from 8 to $9.7 \%$. If the mean heart dose would be $10 \mathrm{~Gy}$ and in the presence of cardiac risk factors, this risk would increase from 8 to $13.5 \%$ [24]. Although, with breath-hold IMPT, the mean heart dose could be reduced to almost zero, the question arises whether all left-sided breast cancer patients will have clinically relevant benefit from proton irradiation. Recently, Langendijk et al. described the so-called modelbased approach, to define which patients could be selected for proton therapy. In this model-based approach, the estimated benefit in terms of risk reduction can be obtained by integrating dose differences in prediction models [25]. The excess risk on ischemic heart disease depends on the dose, and the relative increase per Gy is independent of the baseline risk on cardiac events, meaning that the absolute excess risk can be easily estimated by calculating the baseline risk, e.g., the Reynolds score [26], in addition to the mean heart dose.

Apart from the mean heart dose, there are data suggesting that the dose to the LAD coronary artery is most at risk for developing atherosclerosis after left-sided breastconserving radiotherapy due to its anatomical position in 
Table 1 Dose distribution parameters averaged over 20 patients

\begin{tabular}{|c|c|c|c|c|c|c|}
\hline & \multicolumn{4}{|c|}{ Mean (SD) $(n=20)$} & \multicolumn{2}{|l|}{$p$ value } \\
\hline & \multicolumn{2}{|l|}{$\mathrm{BH}$} & \multicolumn{2}{|l|}{ FB } & \multirow{2}{*}{$\begin{array}{l}\text { BH compared to FB, for } \\
\text { both IMPT and IMRT }\end{array}$} & \multirow{2}{*}{$\begin{array}{l}\text { IMPT compared to IMRT, } \\
\text { for both } \mathrm{BH} \text { and } \mathrm{FB}\end{array}$} \\
\hline & IMPT & IMRT & IMPT & IMRT & & \\
\hline \multicolumn{7}{|l|}{ Heart } \\
\hline Mean (Gy) & $0.1(0)$ & $1.5(0.5)$ & $0.2(0.1)$ & $2.7(1.3)$ & $p<0.01$ & $p<0.01$ \\
\hline$D_{\max }(\mathrm{Gy})$ & $0.3(0.3)$ & $8.6(6.2)$ & $1.2(1.7)$ & $24.7(14.7)$ & $p<0.01$ & $p<0.01$ \\
\hline V5 Gy (\%) & $0.1(0.2)$ & $2.5(2.1)$ & $0.5(0.8)$ & $7.4(4.7)$ & $p<0.01$ & $p<0.01$ \\
\hline V20 Gy (\%) & 0 & $0.6(0.8)$ & $0.1(0.2)$ & $3.5(3.0)$ & IMPT: $p=0.02 / \mathrm{IMRT}: p<0.01$ & $p<0.01$ \\
\hline V30 Gy (\%) & 0 & $0.3(0.4)$ & 0 & $2.4(2.3)$ & IMPT: $p=1.80 /$ IMRT: $p<0.01$ & $p<0.01$ \\
\hline \multicolumn{7}{|l|}{ LAD-region } \\
\hline Mean (Gy) & $0.3(0.2)$ & $6.7(5.1)$ & $0.7(0.8)$ & $14.9(9.3)$ & $p<0.01$ & $p<0.01$ \\
\hline$D_{\max }(\mathrm{Gy})$ & $1.8(1.9)$ & $18.8(13.6)$ & $4.5(3.4)$ & $31.4(13.0)$ & $p<0.01$ & $p<0.01$ \\
\hline V5 Gy (\%) & $0.4(0.9)$ & $30.3(25.9)$ & $2.8(5.8)$ & $54.9(25.1)$ & $p<0.01$ & $p<0.01$ \\
\hline V10 Gy (\%) & $0.1(0.3)$ & $18.2(21.5)$ & $0.8(2.7)$ & $42.9(26.6)$ & IMPT: $p=0.04 / \mathrm{IMRT}: p<0.01$ & $p<0.01$ \\
\hline V20 Gy (\%) & 0 & $9.7(15.1)$ & $0.1(0.6)$ & $32.8(27.1)$ & IMPT: $p=\mathbf{0 . 0 6 / I M R T : ~} p<0.01$ & $\mathrm{BH}: p=\mathbf{0 . 2 0} / \mathrm{FB}: p<0.01$ \\
\hline \multicolumn{7}{|l|}{ Bilateral lung } \\
\hline Mean (Gy) & $0.8(0.4)$ & $2.6(0.9)$ & $0.9(0.3)$ & $2.9(1.1)$ & IMPT: $p=\mathbf{0 . 1 0} /$ IMRT: $p<0.02$ & $p<0.01$ \\
\hline$D_{\max }(\mathrm{Gy})$ & $14.6(8.0)$ & $33.4(5.7)$ & $18.0(6.9)$ & $35.5(5.0)$ & $p<0.05$ & $p<0.01$ \\
\hline V5 Gy (\%) & $3.6(1.9)$ & $10.1(3.1)$ & $4.0(1.2)$ & $10.1(3.3)$ & IMPT: $p=\mathbf{0 . 0 8 / I M R T : ~} p=\mathbf{0 . 9 0}$ & $p<0.01$ \\
\hline V20 Gy (\%) & $1.3(0.8)$ & $5.1(2.2)$ & $1.5(0.7)$ & $5.7(2.6)$ & IMPT: $p=\mathbf{0 . 0 6 / I M R T : ~} p=0.03$ & $p<0.01$ \\
\hline \multicolumn{7}{|l|}{ Lung left } \\
\hline Mean (Gy) & $1.5(0.6)$ & $5.4(1.8)$ & $1.6(0.6)$ & $6.1(2.3)$ & IMPT: $p=\mathbf{0 . 2 0} /$ IMRT: $p<0.01$ & $p<0.01$ \\
\hline$D_{\max }(\mathrm{Gy})$ & $23.6(8.2)$ & $37.1(2.8)$ & $27.0(7.0)$ & $38.7(2.3)$ & $p<0.04$ & $p<0.01$ \\
\hline V5 Gy (\%) & $7.1(2.7)$ & $21.4(6.6)$ & $7.7(2.7)$ & $21.9(7.1)$ & IMPT: $p=\mathbf{0 . 1 7} / \mathrm{IMRT}: p=\mathbf{0 . 5 9}$ & $p<0.01$ \\
\hline V20 Gy (\%) & $2.5(1.4)$ & $10.9(4.7)$ & $2.8(1.4)$ & $12.4(5.7)$ & IMPT: $p=0.04 / \mathrm{IMRT}: p=0.02$ & $p<0.01$ \\
\hline \multicolumn{7}{|l|}{ PTVtrim } \\
\hline V95\% $(\%)$ & $99.6(0.32)$ & $97.9(0.15)$ & $99.7(0.19)$ & $97.9(0.18)$ & IMPT: $p=\mathbf{0 . 0 9 / I M R T : ~} p=\mathbf{0 . 7 0}$ & $p<0.01$ \\
\hline V107 \% (\%) & 0 & $0.4(1.0)$ & 0 & $0.2(0.4)$ & IMPT: $p=1.00 / I M R T: ~ p=0.47$ & $p<0.02$ \\
\hline
\end{tabular}

Non-significant data is presented in bold

$B H$ breath-hold, $F B$ free-breathing, IMPT intensity modulated proton therapy, IMRT intensity modulated radiotherapy, V5, V10, V15, V20, V30, and $V 40 G y$ volume receiving $\geq 5,10,15,20,30$, and $40 \mathrm{~Gy}$, respectively, $D_{\max }$ dose encompassing $2 \%$ of the volume. V95\% and V107 \% volume receiving $\geq 95$ and $107 \%$ of the prescribed dose, respectively

relation to the breast [7]. In the current study, the average mean dose to the LAD-region was 6.7 Gy with breath-hold IMRT which could be reduced to 0.3 Gy with breath-hold IMPT. These doses are lower when compared to the mean LAD doses of 20 and 9.4 Gy, without using breath-hold [6, 8]. It should be noted that the methodologies of defining the LAD or LAD-region varied widely among these three studies $[6,8,11]$.

As in most treatment planning comparative studies, some critical notes also apply to this study.

First, set-up errors and geometric changes during radiation treatment are more likely to affect the dose distributions when using IMPT. It should be noted that the effect of range uncertainties and patient breathing motion using IMPT were relatively small, as shown by Ares et al. [21] which is in line with the results of $\mathrm{Xu}$ et al. [27]. However,
Wang et al. compared a passive scattered proton beam with a spot scanning IMPT technique and stated that IMPT is more sensitive for set-up uncertainties and breathing motion [28]. With advanced position verification procedures and adaptive treatment strategies in combination with a breath-hold technique, these uncertainties are expected to be minimized. Furthermore, as pointed out by other authors, set-up errors and range uncertainties need to be accounted for by applying robust IMPT treatment planning techniques rather than by using the traditional CTV-PTV margin concept $[29,30]$.

Second, some authors reported higher skin dose when using protons and, hence, worse cosmetic outcome can be expected. Girodet et al. reported worse cosmetic outcome in accelerated partial breast irradiation (APBI) when using protons. However, they used a single field per treatment 


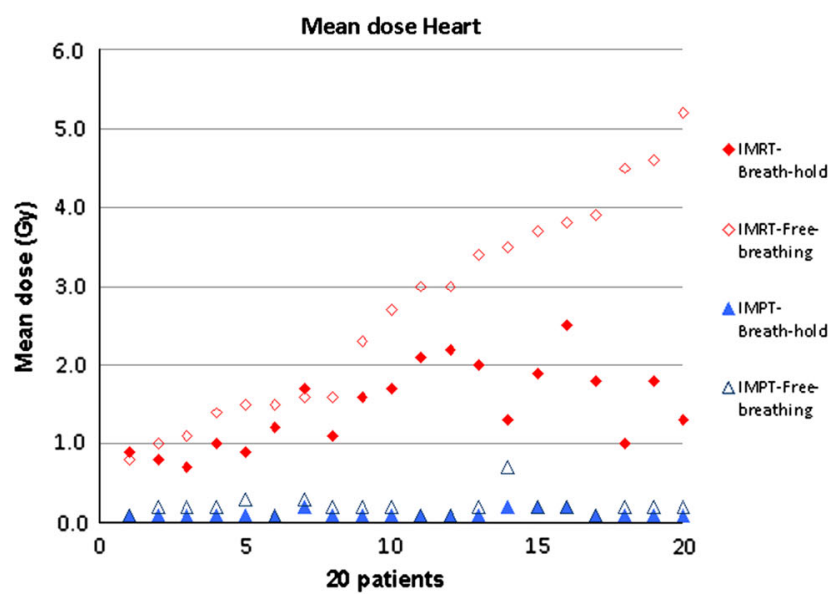

Fig. 2 Left Mean dose administered to the heart. Right Mean dose administered to the LAD-region; both with intensity modulated radiotherapy (IMRT) and intensity modulated proton therapy (IMPT)

and stated that multiple proton beam scanning and advances in patient set-up could result in decreased margins [31]. In our planning comparative study, we were not able to compare the dose to the skin since the treatment planning system used is not able to adequately calculate the dose to the skin. Therefore, the clinical experience when using protons in breast cancer treatment is of importance. Several phase II studies report on the cosmetic results after proton beam therapy $[31,32]$.

Third, for the current study, we decided to use tangential IMRT with $60 \%$ of the dose given with two open tangential fields. Further dose reductions to the heart could be obtained by using IMRT with a larger degree of freedom. However, in most cases this can only be achieved at the expense of dose to other OARS and normal tissue [20,33].

Ares et al. showed that, using proton irradiation, in leftsided breast cancer the dose to the OARs can significantly be reduced when compared to photons [21]. As yet, no planning study has compared proton and photon irradiation in combination with breath-hold in left-sided breast cancer radiotherapy. In most departments, a 3D-CRT photon technique is considered the current standard. However, recently it has been shown that tangential IMRT with breath-hold further reduces the dose to the heart and LAD-region without increasing the dose to other normal tissues [11].

Based on the radiation principles that dose should be "As Low As Reasonably Achievable" (ALARA) there is no doubt that patients will benefit from protons at least to some extent. Due to limited accessibility of proton therapy and higher costs, it will not be feasible to offer protons to all breast cancer patients. A model-based approach will enable the identification of patients who will benefit most from this new technology and thus will ensure a more costeffective use. For all other left-sided breast cancer patients,

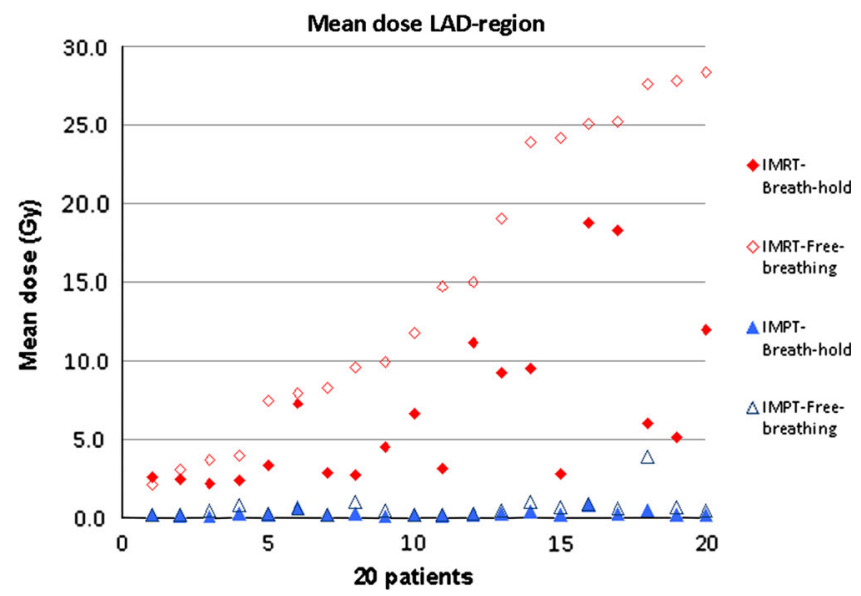

in breath-hold $(\mathrm{BH})$ and free-breathing $(\mathrm{FB})$. The cases were rearranged using the increasing (from left to right) IMRT FB technique values

a tangential IMRT technique with breath-hold can be used to reduce the dose to the heart and LAD-region. In future, it may be possible to make choices based on individual planning comparisons in order to individualize the radiation treatment.

\section{Conclusion}

In left-sided breast cancer irradiation, IMPT is the most promising technique to maximally reduce the dose to heart and LAD-region, even without a breath-hold technique. However, as IMPT for breast cancer is currently not widely available, IMPT should be reserved for patients remaining at high risk for major coronary events.

Acknowledgments We thank T. F. H. Vissers for bibliographical assistance and J. F. D. Bouricius for critically editing this article.

Conflict of interest The authors have nothing to disclose and indicate no potential conflict of interest.

Open Access This article is distributed under the terms of the Creative Commons Attribution Noncommercial License which permits any noncommercial use, distribution, and reproduction in any medium, provided the original author(s) and the source are credited.

\section{References}

1. Early Breast Cancer Trialists' Collaborative Group (EBCTCG), Darby S, McGale P (2011) Effect of radiotherapy after breastn surgery on 10-year recurrence and 15-year breast cancer death: meta-analysis of individual patient data for 10,801 women in 17 randomised trials. Lancet 378(9804):1707-1716

2. Clarke M, Collins R, Darby S et al (2005) Effects of radiotherapy and of differences in the extent of surgery for early breast cancer 
on local recurrence and 15-year survival: an overview of the randomised trials. Lancet 366(9503):2087-2106

3. Kyndi M, Overgaard M, Nielsen HM, Sørensen FB, Knudsen H, Overgaard J (2009) High local recurrence risk is not associated with large survival reduction after postmastectomy radiotherapy in high-risk breast cancer: a subgroup analysis of DBCG 82 b\&c. Radiother Oncol 90(1):74-79

4. Darby SC, McGale P, Taylor CW, Peto R (2005) Long-term mortality from heart disease and lung cancer after radiotherapy for early breast cancer: prospective cohort study of about 300,000 women in US SEER cancer registries. Lancet Oncol 6(8):557-565

5. Hooning MJ, Botma A, Aleman BM et al (2007) Long-term risk of cardiovascular disease in 10-year survivors of breast cancer. J Natl Cancer Inst 99(5):365-375

6. Darby SC, Ewertz M, McGale P et al (2013) Risk of ischemic heart disease in women after radiotherapy for breast cancer. N Engl J Med 368(11):987-998

7. Nilsson G, Holmberg L, Garmo $\mathrm{H}$ et al (2012) Distribution of coronary artery stenosis after radiation for breast cancer. J Clin Oncol 30(4):380-386

8. Taylor CW, Povall JM, McGale P et al (2008) Cardiac dose from tangential breast cancer radiotherapy in the year 2006. Int $\mathrm{J}$ Radiat Oncol Biol Phys 72(2):501-507

9. Schubert LK, Gondi V, Sengbusch E et al (2011) Dosimetric comparison of left-sided whole breast irradiation with 3DCRT, forward-planned IMRT, inverse-planned IMRT, helical tomotherapy, and topotherapy. Radiother Oncol 100(2):241-246

10. Swanson T, Grills IS, Ye H et al (2013) Six-year experience routinely using moderate deep inspiration breath-hold for the reduction of cardiac dose in left-sided breast irradiation for patients with early-stage or locally advanced breast cancer. Am J Clin Oncol 36(1):24-30

11. Mast ME, van Kempen-Harteveld L, Heijenbrok MW et al (2013) Left-sided breast cancer radiotherapy with and without breathhold: does IMRT reduce the cardiac dose even further? Radiother Oncol 108(2):248-253

12. van Asselen B, Schwarz M, van Vliet-Vroegindeweij C, Lebesque JV, Mijnheer BJ, Damen EM (2006) Intensity-modulated radiotherapy of breast cancer using direct aperture optimization. Radiother Oncol 79(2):162-169

13. Levin WP, Kooy H, Loeffler JS, DeLaney TF (2005) Proton beam therapy. Br J Cancer 93:849-854

14. Weber DC, Ares C, Lomax AJ et al (2006) Radiation therapy planning with photons and protons for early and advanced breast cancer: an overview. Radiat Oncol 20(1):22

15. Moon SH, Shin KH, Kim TH et al (2009) Dosimetric comparison of four different external beam partial breast irradiation techniques: three dimensional conformal radiotherapy, intensitymodulated radiotherapy, helical tomotherapy, and proton beam therapy. Radiother Oncol 90(1):66-73

16. Hoppe BS, Flampouri S, Su Z et al (2012) Effective dose reduction to cardiac structures using protons compared with 3DCRT and IMRT in mediastinal Hodgkin lymphoma. Int $\mathbf{J}$ Radiat Oncol Biol Phys 84(2):449-455

17. Mukesh MB, Barnett GC, Wilkinson JS et al (2013) Randomized controlled trial of intensity-modulated radiotherapy for early breast cancer: 5-year results confirm superior overall cosmesis. Am J Clin Oncol 31(36):4488-4497
18. http://www.rtog.org/LinkClick.aspx?fileticket=vZJFhPaBipE\% 3d\&tabid=236; visited 13 June 2014

19. Remouchamps VM, Letts N, Vicini FA et al (2003) Initial clinical experience with moderate deep-inspiration breath-hold using an active breathing control device in the treatment of patients with left-sided breast cancer using external beam radiation therapy. Int J Radiat Oncol Biol Phys 56(3):704-715

20. Mast ME, van der Klein JN, van Geen S et al (2012) Een hartsparende bestralingstechniek bij vrouwen met linkszijdige borstkanker. De resultaten van vier jaar ervaring in Radiotherapiecentrum West. Ned Tijdschr Oncol 9(6):270-276

21. Ares C, Khan S, Macartain AM et al (2010) Postoperative proton radiotherapy for localized and locoregional breast cancer: potential for clinically relevant improvements? Int J Radiat Oncol Biol Phys 76(3):685-697

22. Borst GR, Sonke JJ, den Hollander S et al (2010) Clinical results of image-guided deep inspiration breath-hold breast irradiation. Int J Radiat Oncol Biol Phys 78(5):1345-1351

23. Tan W, Liu D, Xue C et al (2012) Anterior myocardial territory may replace the heart as organ at risk in intensity-modulated radiotherapy for left-sided breast cancer. Int J Radiat Oncol Biol Phys 82(5):1689-1697

24. Duma MN, Molls M, Trott KR (2014) From heart to heart for breast cancer patients: cardiovascular toxicities in breast cancer radiotherapy. Strahlenther Onkol 190(1):5-7

25. Langendijk JA, Lambin P, De Ruysscher D, Widder J, Bos M, Verheij M (2013) Selection of patients for radiotherapy with protons aiming at reduction of side effects: the model-based approach. Radiother Oncol 107(3):267-273

26. Ridker PM, Buring JE, Rifai N, Cook NR (2007) Development and validation of improved algorithms for the assessment of global cardiovascular risk in women: the Reynolds Risk Score. JAMA 297(6):611-619

27. Xu N, Ho MW, Li Z, Morris CG, Medenhall NP (2013) Can proton therapy improve the therapeutic ratio in breast cancer patients at risk for nodal disease? Am J Clin Oncol. doi:10.1097/ COC.0b013e318280d614

28. Wang X, Zhang X, Li X et al (2013) Accelerated partial-breast irradiation using intensity-modulated proton radiotherapy: do uncertainties outweigh potential benefits? $\mathrm{Br} \mathrm{J}$ Radiol 86:1029-1040

29. Unkelbach J, Bortfeld T, Martin BC et al (2009) Reducing the sensitivity of IMPT treatment plans to setup errors and range uncertainties via probabilistic treatment planning. Med Phys 36:149-163

30. Fredriksson A, Forsgren A, Hårdemark B (2011) Minimax optimization for handling range and setup uncertainties in proton therapy. Med Phys 38:1672-1684

31. Galland-Girodet S, Pashtan I, MacDonald SM et al (2014) Longterm cosmetic outcomes and toxicities of proton beam therapy compared with photon-based 3-dimensional conformal accelerated partial-breast irradiation: a phase 1 trial. Int $\mathbf{J}$ Radiat Oncol Biol Phys. doi:10.1016/j.ijrobp.2014.04.008

32. Bush DA, Do S, Lum S et al (2014) Partial breast radiation therapy with proton beam: 5-year results with cosmetic outcomes. Int J Radiat Oncol Biol Phys. doi:10.1016/j.ijrobp.2014.05.1308

33. Borges C, Cunha G, Monteiro-Grillo I, Vaz P, Teixeira N (2014) Comparison of different breast planning techniques and algorithms for radiation therapy treatment. Phys Med 30(2):160-170 